\title{
Lo mío y lo común
}

Mine and common

\section{Gabriel Araújo Pacheco \\ bielpacheco14@hotmail.com}

Graduando em Filosofia pela Universidad

Católica de Córdoba - UCC

\section{Resumen}

Este ensayo tiene el objetivo de, a partir de ideas de algunos filósofos acerca de la vida humana y social - Descartes (1985), Locke (2010), Arendt (2009), Levinas (2000; 2002) -, explicitar la fuerza de la noción de lo "mío"1 en nuestro pensamiento actual y las posibles alternativas para tal paradigma, desde la noción de lo "común"2. Partiendo de los filósofos, y relacionando con lo que nos dice el papa Francisco, respecto de un modo de vida y una economía solidarios, se concluye, por dos experiencias concretas, que la responsabilidad y compromiso son importantes y necesarios. Palabras clave: modernidad; propio; común; responsabilidad; solidaridad.

\begin{abstract}
The objective of this essay is to, starting from the ideas of some philosophers about the human and social life - Descartes (1985), Locke (2010), Arendt (2009), Levinas (2000; 2002) -, specify the strength of the notion of "mine" in our contemporary thought and the possible alternatives to such paradigm, from the notion of "common". Starting from the philosophers, and relating it with what Pope Francis tells us, about a solidary lifestyle and economy, it is concluded, by two concrete experiences, that responsibility and commitment are important and necessary.
\end{abstract}

Keywords: modernity; mine; common; responsibility; solidarity.

\footnotetext{
${ }^{1}$ Las comillas son mías.

${ }^{2}$ Las comillas son mías.

DIGNIDADE RE-VISTA | ISSN2525-698X | 2020 | V. VI | N. 11 | Empreendedorismo Social: rumos e possibilidades para a economia solidária. Pastoral Universitária Anchieta PUC-RIO.
} 


\section{Introduciendo y problematizando}

"Hay un modo de entender la vida y la acción humana que se ha desviado y que contradice la realidad hasta dañarla" (Francisco, 2015, n.101).

Las palabras del Papa Francisco, en la Laudato Si, son hoy una llamada de atención para una realidad que, para nosotros, parece normalizada y justificada, pero que presenta rasgos que necesitan ser revistos. La humanidad, por mucho tiempo, se creyó dueña y propietaria del mundo y, justificada por tales principios, ha construido una lógica en la cual la dominación sobre la naturaleza, sobre los otros, es normal e, incluso, necesaria.

Partimos de la Laudato Si pues en ella Francisco hace aparecer al público, con otra mirada, la que denuncia y pide respuestas, una realidad que interpela (en este caso la crisis ecológica). Nosotros, muchas veces, nos encontramos en el lugar de aquellos que se han acostumbrado y que, por lo tanto, se sienten indiferentes a tal realidad. Pero nuestros tiempos y lo que vemos en ellos piden nuevos rumbos y caminos, soluciones.

Primero, iremos observar algunas características que, gestadas en la Edad Moderna, son muy presentes en la actualidad. Tomamos los pensamientos de los filósofos Descartes (1985) y Locke (2010), para leer a esa mentalidad que nos atraviesa. Después, como alternativas que surgen en el pensamiento contemporáneo del siglo XX, reflexionamos sobre la vida y acción pública en Arendt (2009), el vivir juntos, y sobre el sentido de la responsabilidad que invita a la solidaridad con el otro y los vulnerables, en Levinas (2000; 2002).

El objetivo de este escrito es sensibilizar sobre la importancia y la necesidad de pensar desde otros parámetros a lo económico, puesto que los efectos demuestran la fragilidad y lo peligroso que es una economía tal como pensamos hoy.

\section{Apuntes sobre lo económico}

La alianza entre la economía y la tecnología termina dejando afuera lo que no forme parte de sus intereses inmediatos. (Francisco, 2015, n.54)

Para pensar la propuesta de una nueva economía, es necesario, en algunas palabras, intentar descubrir qué consiste lo económico y para comprender el paradigma tecnocrático en que vivimos, 
como dice Francisco, es esencial analizar esa alianza de la economía en ese juego actual. ¿Qué es lo económico? ¿Qué función y lugar ocupa en la sociedad actual?

La economía es un área del saber relacionada a las necesidades de la casa, a la administración de los bienes y de las riquezas. Si miramos la etimología veremos que la palabra surge de la raíz griega oikos, que quiere decir casa, lo que tiene que ver con lo familiar, lo privado, lo mío. Eso nos dice Pérez (2005, p.72): “oikos puede designar al espacio físico de la casa en una ocasión; en otra, a las propiedades; y en otras, a la familia. Éstos (casa, propiedades y familia) son los componentes del oikós". Es un estudio de aquello que es posesión de uno mismo y de un ámbito familiar y de las relaciones entre tales bienes.

La organización económica gana fuerza a partir de los desarrollos teóricos de la modernidad y, sobre todo, con los logros de la Revolución Industrial, cuando con la gran producción de productos, los bienes se acumulan y se empieza una nueva idea acerca de lo económico, más allá de los límites de la familia griega. De lo familiar, de la casa, hacia la economía del fuera, rasgo del giro del pensamiento económico de nuestros tiempos, marcada por su relación con el mercado y con el capitalismo:

No obstante, el regreso continuo al ideal del oikos y una cierta resistencia a la evolución del concepto de oikonomia hacia las prácticas del mercado, señala que en el centro del pensamiento económico antiguo se hallaba el oikos, y así ha sido hasta el siglo XVIII, coincidiendo con la transformación socioeconómica derivada de la Revolución Industrial... Básicamente, la economía que se ha estudiado, a partir del pensamiento económico actual, es la economía de fuera, es decir, el trabajo remunerado y la producción para el Mercado (Pérez, 2005, p.75).

Vemos el crecimiento y consolidación de tal ciencia económica en los siglos XIX y XX y hoy, sin duda, lo económico tiene un lugar especial, tanto que parece que hasta lo político y lo social pierden fuerza y espacio en las discusiones actuales. En lo económico, la propiedad, lo individual siempre tuvo y tiene un lugar privilegiado. Lo mío, lo poseído, está sobre lo nuestro, lo común y lo compartido. Es un punto a discutir, puesto que el valor de lo político y de lo social también hay sido tema de debates y pensamientos entre los contemporáneos.

Hacemos ahora un breve acercamiento a las ideas de dos pensadores modernos, Descartes (1985) y Locke (2010), que nos ayudaran a entender el espíritu de la modernidad y la mentalidad de nuestros días, marcadamente influenciada por aquél, y después, a partir de las ideas de Levinas (2000; 2002) y Arendt (2009), pensadores contemporáneos, del siglo XX, que proponen otra mirada. El papa Francisco (2015), también por sus planteos actuales, nos ayudará a pensar caminos DIGNIDADE RE-VISTA | ISSN2525-698X | 2020 | V. VI | N. 11 | Empreendedorismo Social: rumos e possibilidades para a economia solidária. Pastoral Universitária Anchieta PUC-RIO. 
y soluciones a una economía que, basada en lo propio, siempre ha tendido hacia el olvido de lo marginal, de los de afuera, excluidos.

\title{
Lo mío: sobre el yo moderno a partir de Descartes y Locke
}

\begin{abstract}
"En la modernidad hubo una gran desmesura antropocéntrica que, con otro ropaje, hoy sigue dañando toda referencia común y todo intento por fortalecer los lazos sociales". (Francisco, 2015, n.116)
\end{abstract}

Cuando, en Filosofía, pensamos en el paso del Medioevo a la Edad Moderna, es imprescindible el francés Rene Descartes (1985) y su pensamiento. El filósofo fue el primero que, aunque con un pie en lo que pensaba la edad anterior, destaca y pone a la luz la centralidad del sujeto. En Descartes la subjetividad, el Yo, pasa a ocupar el centro del mundo, es la medida, la fuente del conocimiento y acreditador de la verdad. Basta, para tener una idea general de su pensamiento, acercarse a sus obras fundamentales: Discurso del Método (Descartes, 2000) y Meditaciones Metafísicas (Descartes, 1985).

La filosofía cartesiana tiene como base la certeza del sujeto: “...habiéndome desembarazado oportunamente de toda clase de preocupaciones, me he procurado un reposo tranquilo en apartada soledad, con el fin de dedicarme en libertad a la destrucción sistemática de mis opiniones" (Descartes, 1985, p.31). Los fundamentos de la tal filosofía son el conocimiento seguro, que se gesta en la soledad del yo, lejos de todo y de todos. Interesante es notar que en Descartes el pensar y conocer es lo que rige todo, hasta el mismo actuar humano: “... me ocupo no de actuar, sino solamente de conocer" (Descartes, 1985, p.34). La primera certeza y verdad ratificada es la famosa frase ego cogito, ergo sum, que será el cimiento sobre lo cuál será construido el edificio filosófico cartesiano y moderno.

Yo soy, yo existo: es manifiesto. Pero, ¿por cuánto tiempo?, Sin duda, en tanto que pienso... Soy, en definitiva, una cosa que piensa. Soy una cosa cierta y existente. (...) ¿Qué soy? Una cosa que piensa, que duda, que conoce, que afirma, que niega, que quiere, que rechaza y que imagina y siente (Descartes, 1985, p.37).

La empresa es audaz y se hace fundante de un nuevo mundo. Es el giro filosófico cartesiano que funda la modernidad filosófica. Como dice Garber (2000, p. 93): "Descartes es uno de los pocos hombres que en la Historia de la Filosofía pueden considerarse como instauradores de una DIGNIDADE RE-VISTA | ISSN2525-698X | 2020 | V. VI | N. 11 | Empreendedorismo Social: rumos e possibilidades para a economia solidária. Pastoral Universitária Anchieta PUC-RIO. 
nueva imagen del mundo, que llamamos modernidad. Desde este punto de vista, su pensamiento debe calificarse como revolucionario".

Otro nombre importante es fundamental para adentrarnos al pensamiento de "lo mío", de lo propio, rasgos de lo moderno, es John Locke, conocido como "el fundador del empirismo moderno y el primer gran teórico del liberalismo" (Várnagy, 2000, p.41). Son muy distintos, Descartes y Locke, en algunos puntos (en la teoría del conocimiento, por ejemplo), pero traducen, de modo similar, lo que piensan los hombres de su tiempo e influyen fuertemente en cómo vamos pensar nosotros, los hombres de hoy. Miremos simplemente la fuerza de las ciencias y del pensamiento liberal actuales, a modo de ejemplo.

Tomemos la noción de la propiedad privada, desde su obra Dos Tratados sobre el gobierno civil, para entender como el sujeto, el yo de la modernidad, está traducido en el modo como Locke lee la dimensión "económica", relacionada a los bienes que uno tiene, por derecho. Ahí están grandes principios de los liberales. "Locke presta enorme atención al tema de la propiedad y elabora su célebre teoría para explicar su origen y valor, para algunos una apología de la moral burguesa y capitalista" (Várnagy, 2000, p.55). Así piensa Locke (2010, p.60): "propiedad es un término polisémico: en sentido amplio y general implica vida, libertad y hacienda (Segundo Tratado 87, 123, 173), y en un sentido más restringido, bienes, el derecho a heredar, y la capacidad de acumular riquezas" (Várnagy, 2000, p.55).

Para el filósofo, la "ley natural está inscripta en el corazón de los hombres" (Locke, 2010, p.17) y esta:

consiste en ciertas reglas de la naturaleza que gobiernan la conducta humana y que pueden ser descubiertas con el uso de la razón. Todos los individuos tienen una racionalidad implantada "por el mismo Dios (Primer Tratado, 86) ... y cuyo más fuerte deseo es el de la auto-preservación (Primer Tratado, 88) ... la vida, la libertad y los bienes son propiedad de toda persona (Segundo Tratado, 87), en tanto son sus derechos irrenunciables (Várnagy, 2000, p.53).

Acá entra en el pensamiento moderno la idea de que lo mío, desde siempre, por derecho natural, es propiedad individual, privada; "la libertad consiste en que cada uno pueda disponer y ordenar, según le plazca, su persona, acciones, posesiones y su propiedad toda". (Locke, 2010, p.60)

En Locke hay una primacía de lo privado y nadie, por la igualdad natural, puede amenazar o perjudicar lo que es de otro. Hay un límite entre lo mío y lo tuyo cuando el asunto es "vida, libertad, 
salud o posesiones" (Locke, 2010, p.12), y eso debe ser respetado y consentido entre todos los miembros de una sociedad. Para eso existe la sociedad, en Locke: "los hombres entran en sociedad para preservar su propiedad" (Locke, 2010, p.211). Lo común está presente en el estado de naturaleza lockeano, pero concomitantemente lo privado ya está, pues cada individuo tiene propiedad sobre su cuerpo y el labor y trabajo que desarrolla por medio de él. Todo era común originalmente. Pero, "aunque todo pertenezca a los hombres en común, cada hombre es propietario de su propia persona, el trabajo de su cuerpo y la labor de sus manos” (Locke, 2010, p.34).

\section{Lo común: la vida común y pública, en Arendt.}

"Hay demasiados intereses particulares y muy fácilmente el interés económico llega a prevalecer sobre el bien común". (Francisco, 2015, n.54)

Los modernos hacen el giro hacia el yo, la intimidad y la subjetividad, olvidándose o poniendo en segundo plan lo social, y la ciencia moderna apunta hacia los cielos, buscando la trascendencia afuera de la Tierra; Arendt hace un análisis: “el propósito del análisis histórico es rastrear en el tiempo la alienación del Mundo Moderno, su doble huida de la Tierra al universo y del mundo al yo" (Arendt, 2009, p.18). En esto está la alienación de la modernidad, que necesita ser superada.

Otro rasgo moderno es la racionalidad. Basta la frase de Hegel que es la síntesis del proyecto de la modernidad: todo lo real es racional y lo racional, real. Arendt indica novedades; "ni pensamiento, ni razón constituyen las características esenciales de la existencia humana” (Arendt, 2009, p.24); es una grande pensadora del siglo XX que ha reflexionado acerca de temáticas, sobretodo sociales y políticas, en un tono crítico con relación a los modernos. Una noción clave de su pensamiento que traemos para contrastar a lo que vamos viendo de los frutos de la modernidad actualmente es la idea de que la sociedad es una pluralidad de individuos, que solo se entiende como plural y en el cual el propio individuo solo es en cuanto participa y aparece en esa esfera de la pluralidad. Los sujetos están en una esfera que es pública y se ubican en ella por la acción y el discurso, por los actos y palabras: "con palabra y acto nos insertamos en el mundo humano; (...) existimos primordialmente como seres que actúan y hablan”. (Arendt, 2009, p.201-206) 
A dicha inserción no nos obliga la necesidad ni nos impulsa la utilidad. Puede estimularse por la presencia de otros cuya compañía deseemos, pero nunca está condicionada por ellos; su impulso surge del comienzo, que se adentró en el mundo cuando nacimos y al que respondemos comenzando algo nuevo por nuestra propia iniciativa. Actuar, en su sentido más general, significa tomar una iniciativa, comenzar (como indica la palabra griega archein, 'comenzar', 'conducir' y finalmente 'gobernar'), poner algo en movimiento (que es el significado original del agere latino) (Arendt, 2009, p.201).

La acción gana un espacio privilegiado en Arendt y, contra la acción en lo mío (veamos Descartes y Locke) típica de los modernos, ahora el actuar es en presencia de otros, se da en la esfera pública. Creemos que la filósofa tiene un mensaje que puede ser profético delante de una sociedad que está marcada por el privado y lo mío (la fuerza de lo económico es señal visible de eso hoy), que es la apertura de lo público y común como fundamental del propio vivir del hombre. Somos en cuanto vivimos con otros. "La esfera de los asuntos humanos, estrictamente hablando, está formada por la trama de las relaciones humanas que existe dondequiera que los hombres viven juntos" (Arendt, 2009, p.207).

\section{La responsabilidad: los vulnerables y la solidaridad en Levinas.}

Ser yo es (...) tener la identidad como contenido. El yo no es un ser que permanece siempre el mismo, sino el ser cuyo existir consiste en identificarse, en recobrar su identidad a través de todo lo que le acontece. Es la identidad por excelencia, la obra original de la identificación. El Yo es idéntico hasta en sus alteraciones. Las representa y las piensa. La identidad universal en la que lo heterogéneo puede ser abarcado tiene el esqueleto de un sujeto, de la primera persona. Pensamiento universal, es un "yo pienso. (Levinas, 2002, p.60-62)

Leemos a otro filósofo contemporáneo, Levinas, que desde la crítica al Yo moderno abre la posibilidad de pensar a partir de la alteridad. Lo traigo pues, por su pensamiento, es posible indagar sobre un modo de vivir, con otros, que es basado en la responsabilidad y en un compromiso que es respuesta hacia el Otro. "Levinas plantea un giro radical respecto a la tradición filosófica al colocar como fundamento de la solidaridad y la justicia la vulnerabilidad del sujeto" (Palacio, 2015, p.33).

En el mundo de hoy es visible los efectos de una vulnerabilidad que se encarna en rostros concretos: esta vida vulnerable de los márgenes, que nos dice Palacio: "en los márgenes, se encuentran zonas grises del derecho y la ética colindando con la situación concreta de la vida vulnerable" (Palacio, 2018, p.227). Delante del grito y de la llamada que nos hace lo concreto, nuestra tendencia, como hijos de la modernidad que somos, puede ser la indiferencia: “el temor 
nos puede encerrar en un individualismo auto-referenciado e indiferente ante la vulnerabilidad de los otros, ahondando así aún más el proceso de desligamiento social” (Palacio, 2015, p.32).

Levinas (Palacio, 2015) propone pensar al sujeto desde otra perspectiva, desde la vulnerabilidad que nos es propia, a todos los humanos, no desde la omnipotencia del yo pienso moderno. "La humanidad del hombre, la subjetividad, es una responsabilidad por los otros, una vulnerabilidad extrema" (Palacio, 2015, p.34). Antes de pensar, anterior a nuestras razones, somos seres que sentimos, sujetos éticos, capaces de afectarse y de responder: "antes que, por la razón, estrategia, cálculo y astucia, debemos dejarnos llevar por el impulso ético de la emoción y la afección de la propia vulnerabilidad que nos provoca el rostro desnudo del otro" (Palacio, 2015, p.44). El Otro, "el rostro del prójimo significa para mí una responsabilidad irrecusable que antecede a todo pacto, a todo contrato" (Palacio, 2015, p.34).

Observamos una diferencia con lo que decía Locke, donde lo mío viene en primero lugar y el prójimo, lo que está fuera de lo mío, puede ser un riesgo o un peligro. Para eso están los pactos y los contratos, para defender lo privado. Levinas (2000; 2002) cambia la lógica: primero el Otro, después Yo. "En oposición al sujeto fuerte de la filosofía de la conciencia, Levinas opone un sujeto fisurado, herido, despojado de su etnocentrismo y de su egoísmo; sujeto partido en el núcleo de su identidad por la alteridad del Otro" (Palacio, 2015, p.38).

Un hecho importante que debemos considerar, ya que los hechos se dicen y nos interpelan a algo, es el tema de la nítida desigualdad en nuestras sociedades, muchas veces causadas por un sistema económico que mata. Delante del dolor, de los gritos, de las necesidades de los demás es esencial asumir una responsabilidad, dejarse afectar y salir de aquello que es lo mío hacia lo nuestro, la vida que compartimos entre todos. La vida es mayor que cualquier propiedad o bien material que podemos tener, mi vida y la vida del nosotros. "La responsabilidad no es un simple atributo de la subjetividad. La subjetividad no es un para sí; es, una vez más, inicialmente para otro" (Levinas, 2000, p.80).

se trata de decir la identidad misma del yo humano a partir de la responsabilidad, es decir, a partir de esa posición o de esa deposición del yo soberano en la conciencia de sí, deposición que, precisamente, es su responsabilidad para con el otro. La responsabilidad es lo que, de manera exclusiva, me incumbe y que, humanamente, no puedo rechazar. Esa carga es una suprema dignidad del único. Yo no intercambiable, soy yo en la sola medida en que soy responsable. Yo puedo sustituir a todos, pero nadie puede sustituirme a mí. Tal es mi identidad inalienable de sujeto. En ese sentido preciso es en el que Dostoievski dice: «Todos somos responsables de todo y de todos ante todos, y yo más que todos los otros» (Levinas, 2000, p.85). 
Pensar en una economía que sea solidaria es ir en contra lo dominante y una lógica de siglos. Pero partir desde el sujeto, de cada uno, para rescatar la característica ética fundamental de la responsabilidad por otros es fundamental si deseamos construir relaciones solidarias. La solidaridad es la salida de si, el yo que, sin esperar la recíproca, está preocupado y actúa en favor de la vida amenazada. ¡Solidarizarse es hacerse más humano!

\section{Pensando lo concreto: Barranca Yaco y Amazonía, dos casos y una misma cuestión}

En las condiciones actuales de la sociedad mundial, donde hay tantas inequidades y cada vez son más las personas descartables, privadas de derechos humanos básicos, el principio del bien común se convierte inmediatamente, como lógica e ineludible consecuencia, en un llamado a la solidaridad y en una opción preferencial por los más pobres. (Francisco, 2015, n.158)

Concluimos partiendo de la motivación que me hace reflexionar y dar vueltas con eso en la cabeza. Conclusión desde la que pensé en empezar este ensayo. La Filosofía, campo a que vengo dedicando tiempo y del cual salen mis lecturas de hoy en día, ilumina a situaciones concretas que, en estos tiempos, de algún modo, tengo contacto, directo o indirecto.

Un caso más local y que me cuestiona son mis visitas a un barrio de la ciudad de Córdoba, en Argentina, donde voy con algunos jóvenes y adultos de un Centro Juvenil de la Compañía de Jesús y de una Parroquia que atiende al barrio. La pobreza, la vulnerabilidad se muestra con toda su desnudez. Allá, donde la propiedad, tal como la entendemos, no existe y donde la ley no está (no protege, ni promueve, ni “controla”...). Un barrio donde puedo escuchar, sin mediaciones, lo que la realidad me tiene que decir. "Mis hijos valen mucho para mí... Hago todo por ellos, aunque sea muy poco lo que tengo"; "no puedo esperar del gobierno una ayuda ni pensar en guardar la poca plata que tengo... llegó el invierno y tuve que levantar las paredes y cubrir con eso. Es muy precaria esa "casa" que hice, pero fue lo que pude hacer para pasar mejor el invierno con mis hijos..."; "ustedes están distribuyendo esas bolsas? Tengo unos paquetes de yerba-mate que creo que serán de mucho provecho a los que aquí en el barrio necesiten más que yo"3.

Puse algunas de las voces de la gente, de esas voces que no necesitan explicaciones. Hablan por sí y afectan a los que las quiere escuchar. Es un caso local que traduce la perversidad de un

\footnotetext{
${ }^{3}$ Frases escuchadas en el barrio.

DIGNIDADE RE-VISTA | ISSN2525-698X | 2020 | V. VI | N. 11 | Empreendedorismo Social: rumos e possibilidades para a economia solidária. Pastoral Universitária Anchieta PUC-RIO.
} 
modelo económico que es injusto y, a la vez, revela un corazón lleno de solidaridad y con un sentido común que hacen más plena tal comunidad. Los problemas están, y necesitan ser resueltos.

Un caso más general y visible que, desde mucho tiempo, me interpela, es el caso de la Amazonia, que sufre los efectos del problema del egoísmo y de la dominación del hombre. El Papa Francisco, al convocar el Sínodo de la Amazonia y al escribir el documento Querida Amazonia, tiene conciencia y nos alerta sobre el tema:

Estamos siendo afectados por los madereros, ganaderos y otros terceros. Amenazados por actores económicos que implementan un modelo ajeno en nuestros territorios. Las empresas madereras entran en el territorio para explotar el bosque, nosotros cuidamos el bosque para nuestros hijos, tenemos la carne, pesca, remedios vegetales, árboles frutales [...]. La construcción de hidroeléctricas y el proyecto de hidrovías impacta sobre el río y sobre los territorios [...]. Somos una región de territorios robados». (Francisco, 2020, n.11)

Amazonia es un territorio con pueblos que sufren. Los pobres y los originarios se encuentran amenazados y por su grito piden la ayuda de aquellos que, por el poder que tienen, pueden protegerlos y garantizar una vida digna, que antes tenían y que ahora ya no la pueden vivir plenamente. Están en una lucha constante para conservar sus principios. Esos pueblos también pueden enseñarnos el sentido de la fraternidad y de la comunidad, valores que los modernos o influidos por ellos hemos olvidado o relativizado.

La lucha social implica una capacidad de fraternidad, un espíritu de comunión humana. Entonces, sin disminuir la importancia de la libertad personal, se evidencia que los pueblos originarios de la Amazonia tienen un fuerte sentido comunitario. Todo se comparte, los espacios privados - típicos de la modernidad - son mínimos. La vida es un camino comunitario donde las tareas y las responsabilidades se dividen y se comparten en función del bien común. No hay lugar para la idea de individuo desligado de la comunidad o de su territorio. Esas relaciones humanas están impregnadas por la naturaleza circundante, porque ellos la sienten y perciben como una realidad que integra su sociedad y su cultura, como una prolongación de su cuerpo personal, familiar y grupal (Francisco, 2020, n.20).

Dos casos concretos que revelan: primero, que lo que dice Descartes, Locke y toda una cantidad de pensadores, se encarna y es visible hoy en día. El individualismo y el egoísmo no pueden tener la última palabra; segundo, que una solución es posible; Arendt, Levinas y tantos otros nos ayudan a considerar tal posibilidad: la defensa de lo común, de lo nuestro, frente al imperio de lo mío y de lo propio. Además, el último objetivo es destacar que el verdadero clamor es concreto: de los pobres que piden justicia, de las personas, de los humanos que comparten con 


\section{DIGNIDADE}

nosotros condición y vida. Sobre una economía más solidaria y justa, tenemos mucho que aprender con los que "menos saben, menos tienen" (y los que los más sabios son). 


\section{Referencias bibliográficas}

ARENDT, H. La condición humana. Buenos Aires: Paidós, 2009.

DESCARTES, R. Discurso del Método. Madrid: Gredos, 2011. Meditaciones Metafísicas. Barcelona: Orbis, 1985.

GARBER, D. "René Descartes". In: Enciclopedia Iberoamericana de Filosofía, del Renacimiento a la Ilustración II, v.21. Madrid: Trotta, 2000, p.93-119.

LEVINAS, E. Ética e Infinito. Madrid: A. Machado Libros S.A., 2000.

LEVINAS, E. Totalidad e Infinito. Ensayo sobre la exterioridad. Salamanca: Sígueme, 2002.

LOCKE, J. Segundo Tratado sobre el Gobierno Civil. Madrid: Tecnos, 2010.

PALACIO, M. "La vulnerabilidad fundando la ética de la solidaridad y la justicia". In: Análisis. Revista de Investigación Filosófica, v.II, n.1, p.29-47, 2015.

PALACIO, M. La vulnerabilidad de la vida y los márgenes de la justicia. Espacios de paz: lectura intercultural de un signo de estos tiempos. Buenos Aires: Ágape, 2018. p.217-228.

PAPA FRANCISCO. Laudato Si. Carta Encíclica sobre el cuidado de la casa. Roma, 2015.

PAPA FRANCISCO. Querida Amazonia, Exhortación apostólica post-sinodal. Roma, 2020.

PÉREZ, M. D. M. "Oikos y oikonomia: El análisis de las unidades domésticas de producción y reproducción en el estudio de la Economía antigua". In: Gerión. Revista De Historia Antigua, n.22 (1), 2005. p.61-79. Disponible https://revistas.ucm.es/index.php/GERI/article/view/GERI0404120061A Acceso en: 09 nov 2020.

VÁRNAGY, T. "El pensamiento político de John Locke y el surgimiento del liberalismo". In: $L a$ filosofía política moderna. De Hobbes a Marx. Buenos Aires: CLACSO, 2000. p.41-76. 